\title{
Mechanistic models enable the rational use of in vitro drug-target binding kinetics for better drug effects in patients
}

\author{
Wilhelmus EA de Witte, Yin Cheong Wong, Indira Nederpelt, Laura $\mathrm{H}$ \\ Heitman, Meindert Danhof, Piet H van der Graaf, Ron AHJ Gilissen \& \\ Elizabeth C.M. de Lange
}

To cite this article: Wilhelmus EA de Witte, Yin Cheong Wong, Indira Nederpelt, Laura H Heitman, Meindert Danhof, Piet H van der Graaf, Ron AHJ Gilissen \& Elizabeth C.M. de Lange (2016) Mechanistic models enable the rational use of in vitro drug-target binding kinetics for better drug effects in patients, Expert Opinion on Drug Discovery, 11:1, 45-63, DOI: $\underline{10.1517 / 17460441.2016 .1100163}$

To link to this article: http://dx.doi.org/10.1517/17460441.2016.1100163

曲 Published online: 20 Oct 2015.

III Article views: 636

View Crossmark data $־$
Submit your article to this journal ¿

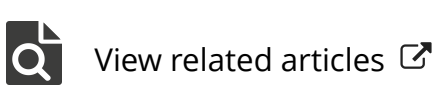

Citing articles: 3 View citing articles $₫$ 


\section{EXPERT OPINION}

1. Introduction

2. In vitro methodological approaches to measure binding kinetics

4. Comparison of in vitro and in/ ex vivo measurements of binding kinetics

5. Missing links in the translation between in vitro and in vivo binding kinetics

6. Conclusion

7. Expert opinion
Taylor \& Francis Taylor \& Francis Group

\section{Mechanistic models enable the} rational use of in vitro drug-target binding kinetics for better drug effects in patients

\author{
Wilhelmus EA de Witte, Yin Cheong Wong, Indira Nederpelt, \\ Laura H Heitman, Meindert Danhof, Piet H van der Graaf, Ron AHJ Gilissen \\ \& Elizabeth C.M. de Lange ${ }^{\dagger}$ \\ ${ }^{\dagger}$ Division of Pharmacology, Leiden Academic Centre for Drug Research, Leiden University, Leiden, \\ The Netherlands
}

Introduction: Drug-target binding kinetics are major determinants of the time course of drug action for several drugs, as clearly described for the irreversible binders omeprazole and aspirin. This supports the increasing interest to incorporate newly developed high-throughput assays for drugtarget binding kinetics in drug discovery. A meaningful application of in vitro drug-target binding kinetics in drug discovery requires insight into the relation between in vivo drug effect and in vitro measured drug-target binding kinetics.

Areas covered: In this review, the authors discuss both the relation between in vitro and in vivo measured binding kinetics and the relation between in vivo binding kinetics, target occupancy and effect profiles.

Expert opinion: More scientific evidence is required for the rational selection and development of drug-candidates on the basis of in vitro estimates of drug-target binding kinetics. To elucidate the value of in vitro binding kinetics measurements, it is necessary to obtain information on systemspecific properties which influence the kinetics of target occupancy and drug effect. Mathematical integration of this information enables the identification of drug-specific properties which lead to optimal target occupancy and drug effect in patients.

Keywords: drug-target binding kinetics, endogenous competition, in vitro, in vivo, mechanistic, nonspecific binding, patients, PKPD modeling, rebinding, target turnover

Expert Opin. Drug Discov. (2016) 11(1):45-63

\section{Introduction}

The rates of drug-target association and dissociation are essential determinants of the time course of target binding and drug effect. This is most clearly illustrated by the irreversible binders aspirin and omeprazole, which have shown a long-lasting effect in clinical practice.[1-3] Numerous other examples confirm that drug-target binding kinetics are important drug characteristics, as reviewed by others.[4-6]

The relevance of drug-target binding arises from their connecting role between pharmacokinetics and pharmacodynamics. More precisely, for a given drug concentration profile, the kinetics of drug-target binding determine the time course of target occupancy and thus the time course of drug effect. The basic concepts of target equilibration kinetics are well established. The simplest mechanism to describe drug-target binding is depicted in Equation 1: 


\section{Article highlights.}

- New in vitro methods for measurement of drug-target binding kinetics to enable their use in drug discovery.

- Various in vitro and in vivo measurement methods of binding kinetics are available, but their validity is not well defined.

- Compared dissociation rate constant values from in vitro and in vivo measurements reveal inconsistent discrepancies.

- These discrepancies can be expected from the unaccounted presence of other kinetic factors in both in vivo and in vitro experiments.

- Mechanistic models can account for these processes to analyze and predict the impact of drug-target binding kinetics on drug effect.

This box summarizes key points contained in the article.

$$
T+L \underset{k_{\mathrm{on}}}{\stackrel{k_{\mathrm{off}}}{\rightleftarrows}} T L
$$

in which $T$ is the target concentration, $L$ is the ligand concentration, $k_{\mathrm{on}}$ is the second-order association rate constant and $\mathrm{k}_{\mathrm{off}}$ is the first-order dissociation rate constant. However, more complex mechanisms have been described in which target activation and G-protein coupled receptors (GPCRs) are incorporated.[5,7] Affinity, the ratio of the dissociation and association rate constants $\left(K_{\mathrm{D}}=k_{\mathrm{off}} / k_{\mathrm{on}}\right)$, is related to binding kinetics, but informs only on the extent of binding at equilibrium and gives no information on the required time to reach a new equilibrium.

The important role of drug-target binding kinetics as a determinant of target occupancy profiles has been known for long, and both in vitro and in vivo measurements of association and dissociation kinetics have been reported from the 1980s.[8-10] However, with the development of highthroughput in vitro methods for binding kinetics, such as surface plasmon resonance (SPR), the interest in the use of binding kinetics in drug discovery has been rising in the past 10 years. This has also led to the development of structurekinetics relationships (SKRs) for some drug classes.[11,12] The recent attention for binding kinetics in drug discovery focuses mostly on the drug-target dissociation rate, since a slow dissociation rate is expected to give a prolonged duration of drug action and improved efficacy.[5,6,13-16]

While most recent publications express an expected benefit of incorporating drug-target binding kinetics in drug discovery, more critical studies have also been published. On the basis of basic pharmacokinetic/pharmacodynamic simulations, Dahl and Akerud indicated that the relevance of binding kinetics in drug treatment depends on a drug's pharmacokinetics.[15] Several other studies have indicated that multiple other physiological processes can influence the impact of drug-target binding kinetics on drug effect, including endogenous competition, diffusion-limited binding and signal transduction.[17-19] While these simulations might contain oversimplifications and cannot be applied to all cases of drug treatment, it is important to realize that the impact of drug-target binding kinetics on drug action depends on multiple kinetic processes in the human body.

To incorporate the role of drug-target binding kinetics in this complexity of kinetic processes, mathematical models have been developed to describe and predict the time profile of drug effects for several drugs and targets.[20-26] These models have been used to estimate drug-target binding kinetics on basis of pharmacokinetic and pharmacodynamic data, which support the relevance of drug-target binding kinetics for drug action.

In summary, the available literature indicates a growing interest in the application of screening techniques for binding kinetics in drug discovery and a context dependency for the impact of drug-target binding kinetics on drug effect. This poses the question under which conditions the in vitro screening of binding kinetics would further drug discovery and development. To answer this question, this review aims to investigate the value of in vitro binding kinetics measurements for the prediction of in vivo target occupancy and drug effect, using available literature with emphasis on two questions:

- What is the relation between in vitro measured binding kinetics and in vivo measured binding kinetics?

- To what extent do binding kinetics contribute to target occupancy and drug effect profiles in vivo?

To that end, first, the available methods to measure drugtarget binding kinetics both in vitro and in vivo are addressed and discussed. Second, we discuss to what extent the estimates of these in vitro and in vivo methods provide comparable results, and what experimental conditions are required to enable translation of in vitro to in vivo binding kinetics. Third, we discuss binding kinetics in a broader perspective, i.e. in the context of the other determinants of target occupancy and drug effect. Finally, the integration of all kinetic processes is discussed, as well as their implementation in the various phases of drug discovery and development.

\section{In vitro methodological approaches to measure binding kinetics}

\subsection{Labeled-ligand assays}

Various methods are available to determine in vitro kinetic binding parameters of compounds of interest at their respective target. In this review, we will use "ligand" to refer to compounds of interest (either labeled or unlabeled) and we will use "tracer" to refer to labeled or unlabeled compounds with known binding characteristics intended to inform about the binding of compounds of interest. The methods as discussed below are summarized in Table 1. 


\subsubsection{Radiolabel-based assays}

The most commonly used and straightforward method to characterize target binding is the use of radioligand binding assays. These assays use a radiolabeled ligand and can directly measure the association and dissociation rates of the radiolabeled ligand. In addition to traditional association and dissociation experiments, other kinetic radiolabel-based binding assays such as a competition association assay are emerging. This type of assay is an indirect assay based on a theoretical model developed by Motulsky and Mahan in 1984 by which one can quantitatively determine the binding kinetics of unlabeled ligands in a competitive assay using only one radiotracer.[16,27-29] The competition association assay can also be used in a higher-throughput fashion with the recently developed dual-point competition association assay. Only two time points are selected here to measure radiotracer binding; the ratio of binding at both time points gives a qualitative measure of the ligands' dissociation kinetics. This makes this simplified assay a suitable method for screening potential drug candidates with favorable dissociation kinetics.[30]

\subsubsection{Fluorescent label-based assays}

Similarly, instead of using a radiolabeled tracer in a competition assay the tracer can also be fluorescently labeled and used in homogeneous time-resolved fluorescence (HTRF) assays. Similar to the radioligand competition assay, only one fluorescently labeled tracer is required and the binding kinetics of competitive ligands can be determined in an indirect fashion. This method is homogeneous since it requires no physical separation of bound and free ligand which enables continuous measurements and increases the throughput. HTRF assays are successfully applied in the determination of binding kinetics of dopamine $\mathrm{D}_{2}$ receptor antagonist spiperone [31] and more recently for histamine $\mathrm{H}_{1}$ receptor ligands [32] and GnRH receptor agonists.[33] Of note, in addition to a fluorescently labeled tracer a fluorescently labeled receptor is needed for this method, as opposed to wild-type receptors for radioligand and radiotracer binding.

\subsection{Label-free assays}

Several label-free methods can be applied for kinetic target binding measurements without the need of a labeled ligand or labeled tracer.

\subsubsection{Surface plasmon resonance}

The most instilled label-free measurement is SPR spectroscopy.[34] This method has the potential to be mediumthroughput and the capability to measure real-time quantitative binding kinetics of ligands for membrane proteins using relatively small quantities of protein. The traditional SPR method needs one immobilized binding component on a coated gold sensor chip during which the ligand in solution is flowed over the sensor chip. This induces a real-time 
change in the refractive index on the sensor surface which is linear to the number of molecules bound.[34-36]

\subsubsection{Acoustic wave biosensor}

Another label-free technology is the surface acoustic wave biosensor.[37] This methodology captures real-time mass changes on the surface, which result in a shifted phase and/ or changed amplitude of a sound wave signal.[38] A disadvantage of these biophysical approaches for $G$ proteincoupled receptors is that these receptors are integral membrane proteins that rapidly disintegrate when taken out of their natural environment, which is a prerequisite for these approaches. However, recent advances are made to overcome this problem.[34]

\subsection{Functional assays}

Another way to determine drug-target binding kinetics is by use of functional assays. These assays provide an indirect measurement of binding kinetics by characterizing the time profile of drug effect. Although the use of functional assays is generally limited due to the indirect nature of these measurements, functional assays are valuable for the measurement of enzyme binding kinetics because of the direct relation between enzymatic product generation rates and enzyme inhibitor binding. Functional assays can be carried out in two different settings, either by resembling the classical "organ bath" experiment or by washout experiments.

\subsubsection{Organ bath}

An organ bath experiment is only suitable to qualitatively examine binding kinetics of antagonists and requires preincubation of cells/tissues with antagonists prior their challenge with an agonist. With this method the distinction between so-called surmountable and insurmountable antagonists can be made, where the level of insurmountability by an antagonist is related to its receptor dissociation kinetics. $[18,39,40]$

\subsubsection{Washout}

Functional washout experiments are suitable for predicting binding kinetics of both agonists and antagonists. In these types of experiments, the rate of decrease in effect after removal of the free ligand by repeated washing (washout) is measured. Agonists with fast dissociation kinetics will readily wash out and will show a right-ward shift in their potency, whereas agonists with slow dissociation kinetics will show insignificant shifts in their potency, and vice versa for antagonists. It should be stated that control experiments are necessary to confirm that the long-lasting effect of the ligand is due to long target binding versus other effect-prolonging factors (such as exosite binding, membrane partitioning, rebinding or signal transduction).[41-43]

\subsection{In vivo methodological approaches to measure binding kinetics}

\subsubsection{General principle of target occupancy measurements}

To obtain drug-target binding kinetics in vivo, target occupancy and target site concentrations are required. For most in vivo and ex vivo approaches, the target occupancy of a drug is measured indirectly by using a tracer. The administered drug competes at the same target site with the tracer and the reduction in specific binding of the tracer is used to calculate the target occupancy of the drug. The tracer can be an antagonist (more common) or agonist to the target, and can be radiolabeled (more common) or non-radiolabeled. The advantages and disadvantages of each approach are briefly discussed, and the characteristics of each approach are summarized in Table 2 . We focus here mainly on methods which are in use for measurement of binding kinetics in the brain, since most methods have been used primarily for the brain targets.

\subsubsection{Tissue homogenate method with radiolabeled tracer}

The traditional way of measuring CNS target occupancy in preclinical animals is the brain homogenate method. At a predetermined time point after radiotracer administration, the animal is sacrificed and the brain regions of interest (e.g. striatum for $\mathrm{D}_{2}$ receptors) and the reference region (e.g. cerebellum which has relatively low $\mathrm{D}_{2}$ receptor density, for the correction of nonspecific binding of radiotracer to and uptake in brain tissue) are collected. These brain regions are then dissolved in a scintillation cocktail and the drug-induced change in radioactivity of the tracer is measured by a liquid scintillation counter. Literature reports suggest that the target occupancy values obtained by this method are comparable to that obtained by positron emission tomography (PET) imaging.[44] Compared with PET/SPECT (single-photon emission computed tomography) imaging, this method is associated with much lower costs and allows higher throughput in screening different compounds or different doses of a single compound. Nevertheless, since this method involves the terminal use of animals, a continuous target occupancy time profile within the same animal cannot be obtained, and multiple animals are needed for a single target occupancy time profile. Moreover, in addition to the receptors expressed on the membrane surface, intracellular or internalized receptors would also become accessible to the tracer when the tissue is homogenized, which might hamper the accuracy of target occupancy assessment for membrane-bound receptors.[45]

\subsubsection{Tissue homogenate method with non- radiolabeled tracer using $L C / M S$ assays}

The procedures of this method are the same as that with radiolabeled tracer as described above, except that a non-radiolabeled tracer (cold tracer) is administered to the animal 


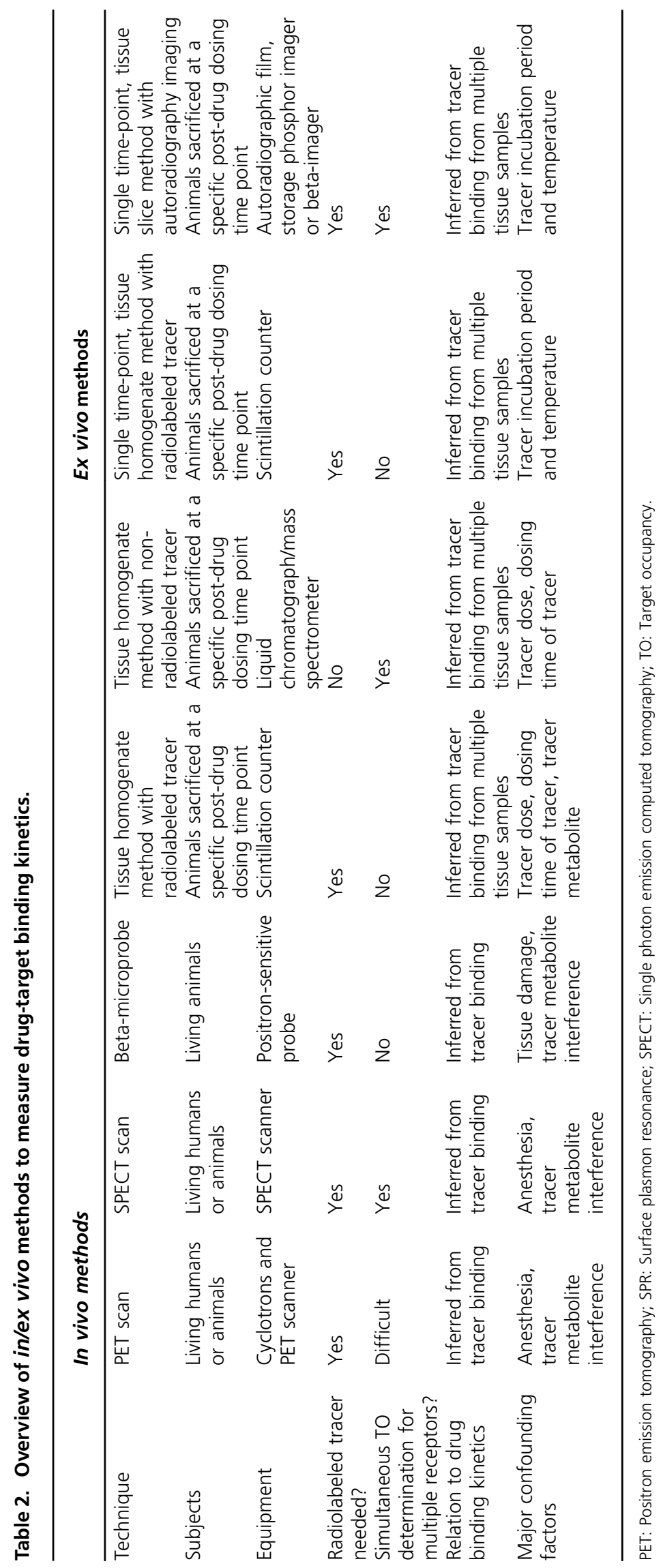


and the absolute amount of the tracer in the brain tissues is quantified by LC/MS. The first report was presented by Phebus and colleagues, in which the drug-induced target occupancy of $\mathrm{D}_{2}$, serotonin $2 \mathrm{~A}$ and NK-1 receptors in rat was quantified using non-radiolabeled tracers.[46] They also demonstrated in rats that for the eight $\mathrm{D}_{2}$-antagonists they had investigated, the doses required to achieve 50\% target occupancy using this LC/MS method (cold raclopride as tracer) are comparable to those using the traditional brain homogenate method $\left(\left[{ }^{3} \mathrm{H}\right]\right.$ raclopride as tracer).[47] This method offers several advantages; first, the parent, intact tracer in the brain tissue can be differentiated from the tracer metabolites, thus increasing the accuracy of tracer quantification. Second, the costs and hazards associated with radioactivity are avoided. Third, it allows separation and quantification of different tracers in one sample, and thus enables the simultaneous assessment of the target occupancy of different receptors.[48]

The greatest concern of this method is the relatively high dose of the tracer that needs to be administered. Since the sensitivity of an LC/MS assay is lower than that of radioactivity counting, a much higher dose of the tracer is administered in order to achieve a quantifiable tissue concentration. This high tracer dose might distort the druginduced target occupancy and might exert pharmacodynamics effects. [49,50]

\subsubsection{PET/SPECT imaging}

PET and SPECT imaging are the most common approaches to measure drug target occupancy in living humans and other primates. After the administration of a very small dose of radiotracer for the desired target, scans are carried out by the PET or SPECT scanner before and after administration of the competing drug. The radioactivity at the region of interest is measured, from which the density of receptors $\left(B_{\max }\right)$ and the radiotracer binding affinity $\left(K_{\mathrm{D}}\right)$ are derived. The ratio of $B_{\max }$ and $K_{\mathrm{D}}$ is termed the binding potential. The target occupancy of the drug is calculated as the percentage reduction in binding potential after drug administration. Binding kinetic parameters $\left(k_{\mathrm{on}}, k_{\mathrm{off}}\right)$ can be derived if the target occupancy and free drug PK at the binding site are available by fitting a mathematical model which describes binding kinetics according to Scheme 1. However, the PET signal arises from the sum of free, specifically and nonspecifically bound radiotracer, and free concentrations cannot be measured at the binding site. Instead of the free drug pharmacokinetics at the binding site, a reference tissue which is similar to the binding site but has no specific binding is commonly used.[51,52] PET/SPECT can be regarded as an in vivo version of autoradiography (discussed in Section 3.2), with inferior spatial resolution but with the advantage that the pharmacokinetics of the tracer can be measured in a single experiment, or even in repeated studies on the same subject.[53] This also provides the possibility to obtain target occupancy values at different time points within the same subject. Over the past decade, there are considerable developments of both PET and SPECT systems with improved spatial resolution designed specifically for small-animal imaging (i.e. microPET and microSPECT).

A limiting factor in longitudinal PET/SPECT measurements is the half-life of the radioactive decay of the tracer (depending on the applied radiolabel), which can limit the duration of the experiment after tracer administration. This limited duration of the imaging decreases the suitability of PET/SPECT for measuring drugs with slow binding kinetics.

One of the main concerns in PET/SPECT is that the anesthesia, applied to immobilize the animals before and during imaging, could hamper the accuracy of target occupancy assessment by, for example, altering the level of neurotransmitters.[54] Moreover, the use of anesthesia might also impose additional experimental variability (e.g. due to variable susceptibility to the anesthetic effect [55]).

Since both the tracer and the drug of interest interact with the same receptor, the observed effect cannot be completely attributed to the drug. Therefore, drug effect measurements are considered less useful, except for studies which are focused on the binding and effect of only the tracer. Depending on the target of interest, the required anesthesia can also interact with drug effects and make their measurement impossible or less useful. Alternatively, the drug effect might be evaluated just before the administration of the tracer (and anesthetics).

\subsubsection{Beta-microprobe}

Another method of measuring a radiotracer in a living animal's brain is the use of a beta-microprobe. The microprobe captures beta/positron emission (similar to the PET detector) and is surgically implanted in the brain structures of interest, allowing in vivo measurement of local radioactivity concentrations within $1-2 \mathrm{~mm}$ from the probe. Reports on the application of beta-microprobe on target occupancy assessment are limited. Good correlations have been reported between in vivo beta-microprobe measurements and ex vivo brain homogenate and in vivo microPET measurements of respectively $\mathrm{D}_{2}$ and $5 \mathrm{HT}_{1 \mathrm{~A}}$ target occupancy in rat brain. [56,57]

The potential advantages of beta-microprobe are that the target occupancy could be measured in awake, non-anesthetized animals and simultaneous assessment of drug-induced changes in behavior is allowed, which are critical for drugs that act on CNS receptors. Nevertheless, the surgical implantation procedures might interfere with the neurochemistry and the pharmacokinetics and pharmacodynamics of the drug and tracer. Implantation of the electrode into the brain would cause mechanical trauma and trigger both acute and chronic tissue responses, and the final outcome depends on factors such as the size, geometry and material of the probe, the insertion method and the period after insertion.[58] Device implantation could also alter the release of neurotransmitters and neural activity.[59] While the 
previously developed beta-microprobes were based on a single pixel scheme that did not provide any spatial information on the radiotracer distribution, [56] a new wireless probe was recently published, which contains 10 submillimeter pixels which allows the analysis of the spatial distribution of the radiotracer within the region of interest in freely moving rats. [60]

\subsection{Ex vivo approaches of target occupancy measurements}

\subsubsection{Tissue homogenate method with radiolabeled}

tracer

While for in vivo methods both the drug and the tracer are administered to the living animals, for ex vivo methods the tracer is added to the collected tissue from the drug-treated animal, and the amount of radiotracer bound to the target in the homogenate is measured by liquid scintillation counting. In this way tracers with unfavorable in vivo characteristics (e.g. slow equilibrium at target tissue, pharmacokinetic variability, etc.) can be used and the costs of developing suitable tracers are reduced and the amount of tracer can be precisely controlled. However, the values of target occupancy obtained by this method are highly dependent on the binding conditions (particularly the time and temperature of tracer incubation) and tend to give an underestimation of drug-induced target occupancy. [61] This is mainly due to the dissociation of the drug from the receptor during the ex vivo tracer incubation and the tissue homogenization step, particularly for those drugs with a fast dissociation rate from the receptor. Therefore, a short incubation time and a radiotracer with a fast association rate are recommended.[62]

\subsubsection{Tissue slice autoradiography imaging}

The procedures of this method are the same as that with tissue homogenate method described above, except that the animal tissue is sectioned into slices and the amount of radiotracer bound to the target is quantified by autoradiography. Unlike tissue homogenate, the tissue slice preparation maintains structural integrity. It offers higher spatial resolution than PET/SPECT imaging and thus allows the investigation of anatomical regions that are small in size. Traditionally, the radioactivity on the slice is captured by autoradiographic film, which requires a long exposure period (weeks) and thus is not considered as an efficient screening method for determining the target occupancy of compounds. [62] The introduction of storage phosphor imaging is a major improvement in ex vivo receptor autoradiography, which shortens the exposure time from weeks to days or even 1 day.[63] An alternative method is to use a beta-imager which uses a highly sensitive gaseous detector of beta particles. This allows the exposure time to be shortened to a few hours.[64]

\section{Comparison of in vitro and in/ex vivo measurements of binding kinetics}

To investigate whether the current in vitro and in vivo measurements of binding kinetics deliver similar or translatable values, we performed a literature survey to identify compounds for which both in vitro and in vivo estimates of target association or dissociation rates were available. Since in vivo estimates are the least available, we started our search with in vivo estimates and continued to search for in vitro estimates of the same compounds. Since the number of compounds for which we could find in vitro and in vivo estimates of their target binding kinetics was very low, we decided to list all estimates we could find and discuss the reliability and comparability of the estimates below. The results of this search are listed in Table 3.

Based on Table 3, we can start to answer our first question:

- What is the relation between in vitro measured binding kinetics and in vivo measured binding kinetics?

From the results in Table 3 , it can be directly seen that the difference between in vitro and in vivo estimates of target dissociation rates can be quite substantial (up to 30fold) and inconsistent (the ratio varies from 0.2 to 31 ). This clearly indicates that the use of in vitro measured target binding kinetics to predict in vivo binding profiles is not straightforward. Apart from the studies in Table 3, another study was published in which no in vivo values for $k_{\mathrm{on}}$ and $k_{\mathrm{off}}$ were included, but in vitro values were used to predict target occupancy profiles of the CRF1 receptor in rats for several antagonists.[23] Although the in vivo results were not highly informative for the identification of the binding kinetics for some compounds in this study, the target occupancy profiles could be predicted reasonably well.

To investigate the origin of the observed difference between in vitro and in vivo binding studies, the experimental details need to be taken into account to identify which results are less reliable or comparable.

\subsection{Temperature}

First, all in vitro estimates of association and dissociation rates which are not obtained at $37^{\circ} \mathrm{C}$ cannot be compared directly to in vivo estimates, since these rates are temperature dependent in a compound-specific manner.[86-88] Therefore entries 3, 9, 12, 13, 17, 18 and 19 in Table 3 cannot be used to compare in vitro and in vivo dissociation rates.

\subsection{Influence of in vivo displacer/competitor dose}

Another important factor in the comparison between in vitro and in vivo estimates of target dissociation rates is the method 


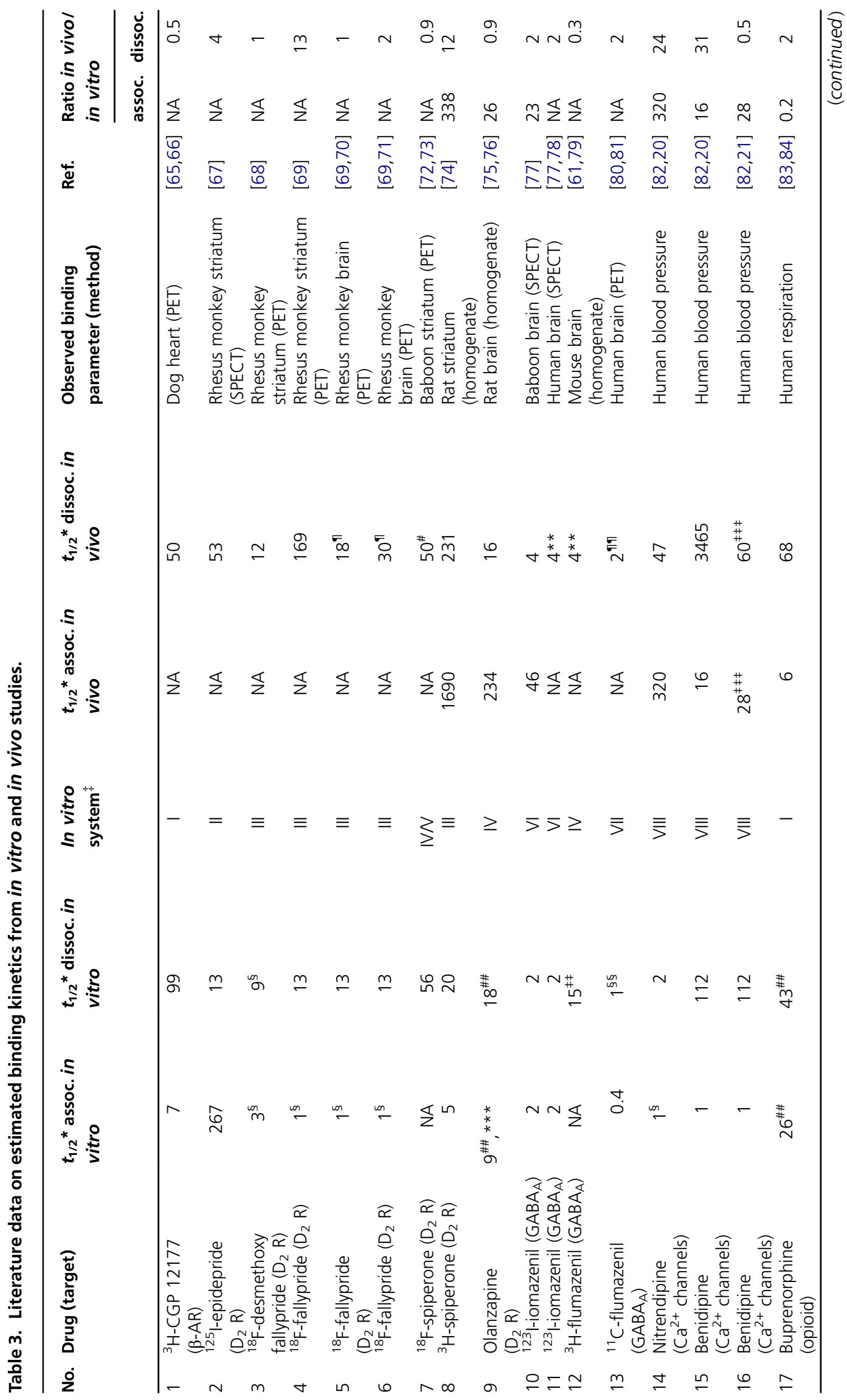




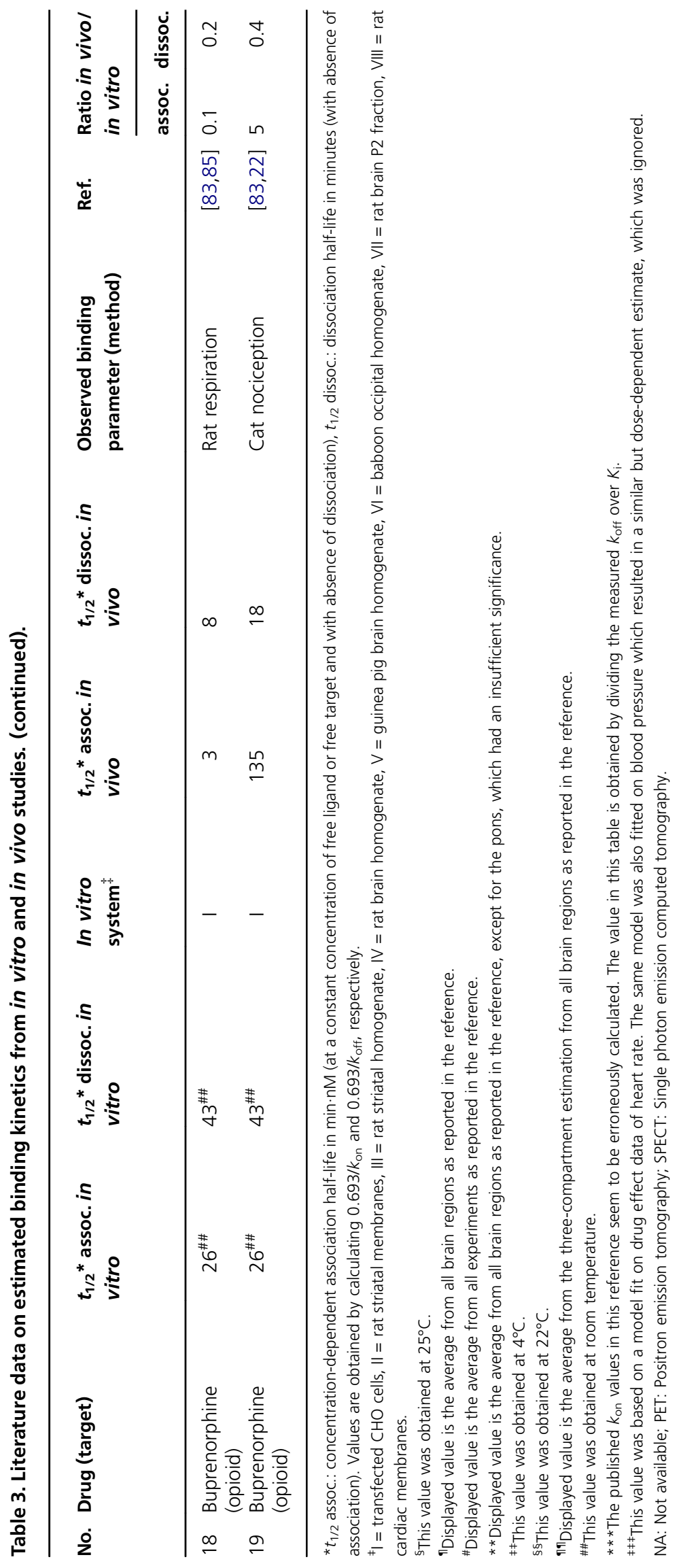


by which the dissociation is induced. Drug-target dissociation can be induced in in vitro studies either by continuous washing, the so-called "infinite dilution" method, or by displacement of the drug by adding an excess of a competing ligand. These methods can give quite different results since washing cannot displace all free ligand molecules and diffusion-limited binding (or "rebinding") can occur. Thus, comparisons between in vitro and in vivo estimates should use the same method of dissociation measurement.[19] However, in the in vivo setting, continuous washing cannot be applied and the amount of competing compound which can be added is limited by its toxicological effects. In the analysis of in vivo drug-target binding studies, computational models can be used to correct for remaining drug concentrations or partial displacement. However, this is often not done and assumptions have to be made about the effect of a displacer dose or of a remaining drug concentration. For entries $1,3,4,8$ and 10 in Table 3, the rationale for the displacer dose was not clear, and model-based analysis was not used. These entries should therefore not be used to compare in vitro and in vivo dissociation rates. For entry 1 , the in vitro experiment did not use either a displacer or continuous washing, which makes it even less appropriate for comparison with the in vivo experiment.

For entries 14-19 in Table 3, the in vivo drug-target binding kinetic parameters are estimated from $\mathrm{PK}$ and $\mathrm{PD}$ data without target occupancy measurements. This makes these estimates indirect and subject to influences of signal transduction kinetics and other factors between PK and PD. Therefore, entries 14-19 cannot be used for a direct comparison of in vitro and in vivo binding kinetic parameters.

\subsection{Most valid comparisons}

To evaluate the difference between in vitro and in vivo estimates of association and dissociation rates, we should only use the most valid comparisons, restricting Table 3 to entries 2, 5, 6, 7 and 11 . Now the ratio between in vitro and in vivo estimates varies between 0.9 and 4 which is considerably better, but based only on four compounds and two targets. Moreover, it should be noted that these entries include only one entry for which the comparison is made with human binding data. Also, all observations in Table 3 originate from GPCRs and, therefore, none of the studies used isolated receptors. One could speculate that the correlation between in vitro and in vivo estimates is better for membrane-bound targets than for soluble targets since the membrane-bound receptors are mostly measured in membrane fractions and therefore retain some of their natural environment, whereas soluble targets can be completely purified. However, the natural exposure of membrane-bound receptors to the differential composition of extracellular and intracellular fluids cannot be reproduced in homogenized in vitro experiments, while the homogeneous environment of soluble targets can be replicated in vitro.

\subsection{Summary}

The amount of available literature data to compare in vitro and in vivo estimates for drug-target dissociation rates in a valid manner is too low to draw general conclusions about the predictive value of the in vitro drug-target dissociation estimates. This is even more so for drug-target association rates. Moreover, differences in experimental approach and conditions and differences in data analysis hamper the comparison of in vitro and in vivo binding kinetics data. These differences include most frequently a difference in temperature (i.e. in vitro experiment not at $37^{\circ} \mathrm{C}$ ), difference in dissociation method (washout vs. displacement) and analysis method (model-based parameter estimation vs. graphical methods). Therefore, the current in vitro estimates of drug-target binding kinetics cannot be translated reliably into in vivo binding kinetics due to a lack of available information on comparability and due to methodological differences between in vitro and in vivo experiments.

\section{Missing links in the translation between in vitro and in vivo binding kinetics}

The differential results that have been observed from in vitro and in vivo studies can be explained by a multitude of differences between the extremely complex in vivo situation and the much more simplified in vitro environment. Possible explanations include factors that are poorly understood, such as the in vivo occurrence of complicated ligand interactions with multiple targets, allosteric binding sites, exosites and subcellular compartments or organelles, but also complex target interactions with other proteins (homo- and heterodimerization), and other cell membrane and intra- and extracellular fluid constituents, such as ions. Moreover, the in vivo three-dimensional structure of multiple cell types is rarely replicated in vitro and unknown contributors to the observed in vivo target binding kinetics cannot be excluded.

However, the following section is focused on the better understood contributors to in vivo target binding kinetics and how these can be accounted for in the design and analysis of both in vitro and in vivo experiments

\subsection{Experimental conditions in in vitro and in vivo studies of binding kinetics}

As described in the previous section, the comparison of in vitro and in vivo binding kinetic parameters is often hampered by differential experimental conditions between in vitro and in vivo studies. We discuss here the most relevant experimental conditions which can hamper the translation between in vitro and in vivo measured binding kinetics. These are: in vitro temperature, in vivo displacement method and the presence of endogenous ligand. 


\subsubsection{Temperature}

One very important in vitro and ex vivo experimental condition is the temperature. Since both drug-target association and dissociation rates are temperature dependent in a compound-specific manner [86-88], translation of binding kinetics from one temperature to another temperature cannot be done unless the temperature dependency has been determined for that specific compound. Moreover, since the target conformation might be temperature dependent as well, the Arrhenius plots of $k_{\text {on }}$ and $k_{\text {off }}$ are not necessarily linear. A few literature examples are available of linear Arrhenius plots for $k_{\text {on }}$ and $k_{\text {off. }}$ [88-90] Therefore, it is highly relevant to obtain in vitro binding parameters at $37^{\circ} \mathrm{C}$, or to obtain a linear Arrhenius plot at lower temperatures.

\subsubsection{Displacer/competitor}

Another condition that may affect translational success is the presence or absence of a displacer/competitor. To account for this, it is necessary to obtain both in vitro and in vivo estimates for $k_{\text {off }}$ in the presence of a displacer. If both experiments are done in the absence of a displacer, translation can still be hampered because of differential diffusion rates and target clustering in the two experiments, leading to different diffusion-limited binding ("rebinding").

\subsubsection{Endogenous ligand}

The presence of an endogenous ligand is also influencing the rates of drug-target association and dissociation. An endogenous ligand can be present both in vitro and in vivo. To enable an accurate in vivo and in vitro estimation of drugtarget $k_{\text {on }}$ and $k_{\text {off }}$ in the presence of an endogenous ligand, the concentration profile over time during the experiment and the binding kinetics of the endogenous ligand need to be known.

\subsection{Integrated analysis of multiple determinants of in vivo target occupancy and drug effect}

In order to use in vitro binding kinetic data to predict in vivo target occupancy and effect kinetics, all kinetic processes which influence the in vivo kinetics of drug effect need to be taken into account (see also Section 7). These include pharmacokinetics, endogenous competition, diffusionlimited binding, nonspecific binding, target turnover and signal transduction. Each of these processes will be discussed in the following sections.

\subsubsection{Pharmacokinetics}

One of the clearest examples for the need to integrate all kinetic processes for the prediction of in vivo target occupancies is the role of pharmacokinetics: If the drug concentration in the human body has a constant profile, an equilibrium situation will be reached and a slow dissociation rate will not prolong the target occupancy anymore. On the basis of a very simple relation between pharmacokinetics and binding kinetics, one can expect a slow dissociation rate to be prolonging target occupancy only when its dissociation rate is slower than its elimination rate (Figure 2, upper panels). $[15,19]$ However, this might be an oversimplification, and other processes need to be integrated as well.[19]

\subsubsection{Endogenous competition}

Another process which is important for the role of binding kinetics is endogenous competition. The presence of a varying concentration of endogenous ligand can make a drug's binding kinetics more important, also when its dissociation half-life does not exceed its plasma elimination half-life (Figure 1).[14,18,91-93] Since endogenous ligands usually have a varying concentration, endogenous competition might be relevant for the binding kinetics of most agonists and antagonists. A hypothesis in this direction was already published by Kapur and Seeman before the recent interest in binding kinetics.[93] In their publication, fast dissociating dopamine antagonists were suggested to be less resistant to dopamine signaling, thereby preventing side effects from over-suppression of dopamine signaling.

\subsubsection{Diffusion-limited binding}

A kinetic process which has got only limited attention for its effect on target occupancy profiles is diffusion-limited binding. If the effective diffusion of a drug around its target is limited, the chance that it will re-associate to its target before diffusing into the tissue (often called "rebinding") will increase and thus the target occupancy will decrease slower than expected from its binding kinetics and tissue concentration. Although the possible significance of diffusion and

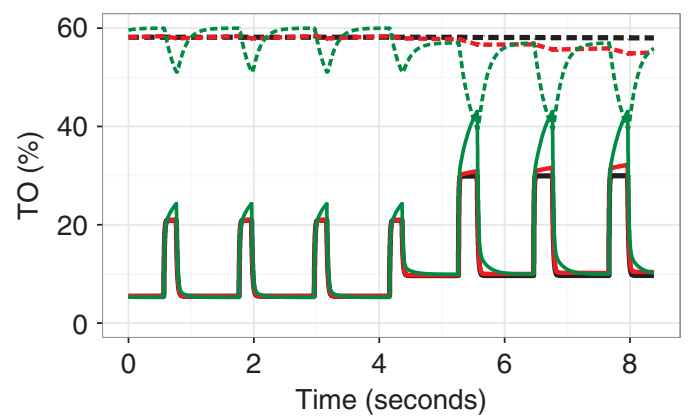

Figure 1. The influence of drug-target binding kinetics on drug (dashed lines) and dopamine (solid lines) target occupancy (TO) is influenced by endogenous competition, as simulated by Vauquelin et al.[18] A constant drug concentration and pulsatile dopamine concentration are used, and the system is allowed to reach equilibrium before $t=0$. The dopamine concentrations rise after $4 \mathrm{~s}$ to represent a high activity period. The drug target dissociation rate $\left(k_{\text {off }}\right)$ changes from $181 \mathrm{~min}^{-1}$ (green) to $6.03 \mathrm{~min}^{-1}$ (red), and $0.181 \min ^{-1}$ (black). 

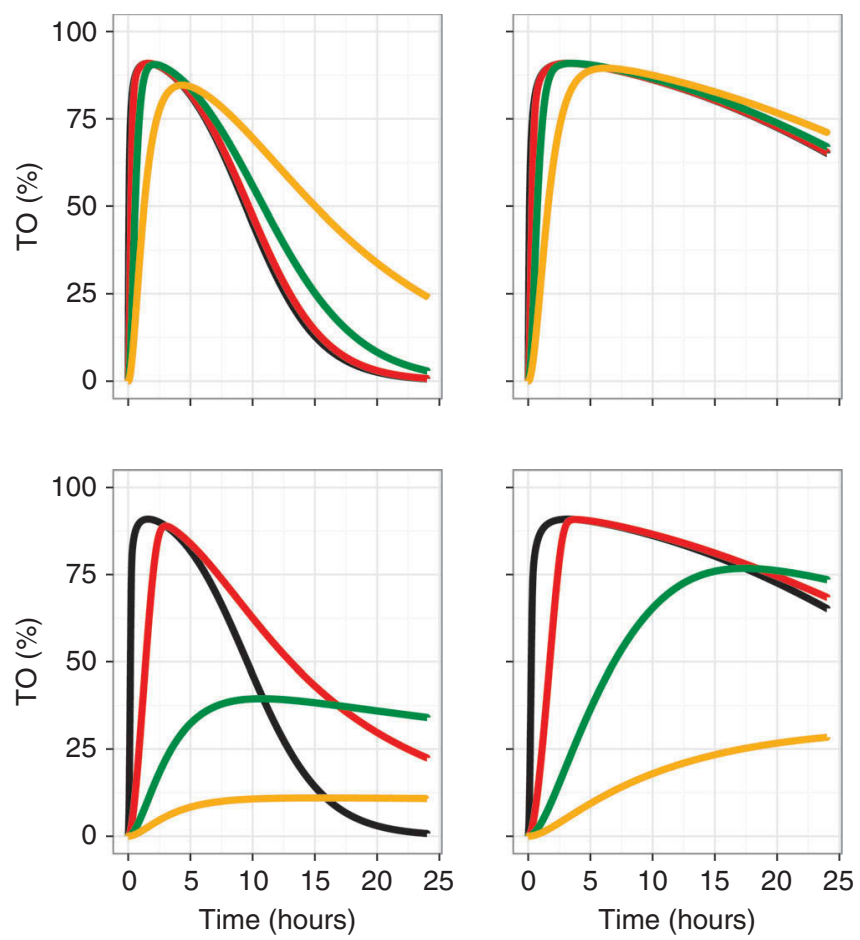

Figure 2. The influence of drug-target binding kinetics on target occupancy (TO) depends on both pharmacokinetics and diffusion-limited binding, as simulated by Vauquelin et al.[19] The drug target dissociation rate $\left(k_{\text {off }}\right)$ changes from $83 \mathrm{~h}^{-1}$ (black) to $2.1 \mathrm{~h}^{-1}$ (red), $0.35 \mathrm{~h}^{-1}$ (green), and $0.087 \mathrm{~h}^{-1}$ (orange). The drug elimination rate constant is $0.35 \mathrm{~h}^{-1}$ for the left panel and $0.087 \mathrm{~h}^{-1}$ for the right panel.

diffusion-limited binding (or "rebinding") has already been indicated in studies with rats, humans and in vitro over three decades ago $[9,10,94]$, there is no general practice of taking this into account in either in vitro or in vivo studies. As reported several times by Vauquelin and his colleagues, based on literature, experimental and theoretical findings, "rebinding" can have a significant impact on the estimated $k_{\text {off }}$ value in in vitro and in vivo studies, and therefore needs to be taken into account in the design and analysis of these studies (Figure 2).[19,41,95-97]

\subsubsection{Nonspecific binding}

Another kinetic process which can influence the profile of target occupancy is nonspecific binding. Nonspecifically bound drug can act as a reservoir which releases drug upon decreasing free drug concentrations, thereby decreasing the effective elimination rate. Moreover, if the release of nonspecifically bound drug is slow, this can become the rate determining factor for the rate of drug elimination from either the plasma or the target tissue (Figure 3). $[98,99]$
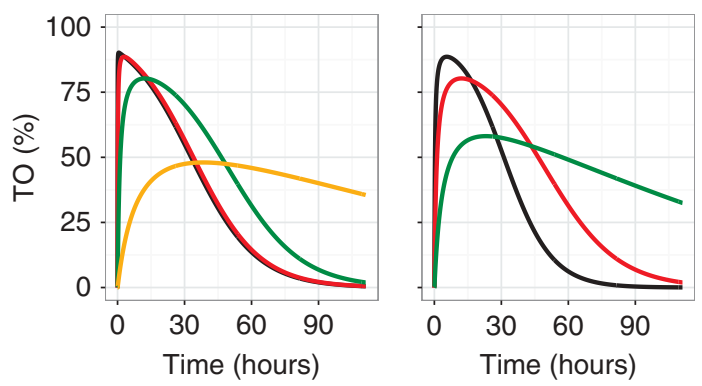

Figure 3. The target occupancy (TO) profile can be influenced by nonspecific binding of the drug, as simulated for lipid and protein binding in the brain by Peletier et al.[98] The drug target dissociation rate constant $\left(k_{\text {off }}\right)$ is $36 \mathrm{~h}^{-1}$ for all lines. For the left panel, the drug-protein dissociation rate constant changes from $1000 \mathrm{~s}^{-1}$ (black) to $100 \mathrm{~s}^{-1}$ (red), $10 \mathrm{~s}^{-1}$ (green), and $1 \mathrm{~s}^{-1}$ (orange). For the right panel, the drug-lipid dissociation rate constant changes from $500 \mathrm{~s}^{-1}$ (black) to $100 \mathrm{~s}^{-1}$ (red) and $20 \mathrm{~s}^{-1}$ (green). The drug-protein and the drug-lipid affinity change in the same way as the dissociation rate constants, since both drug-protein and drug-lipid association rate constants remain unchanged.

\subsubsection{Target turnover}

The rate of target synthesis and degradation can also influence the profile of target occupancy, since the breakdown of occupied target and synthesis of new (unoccupied) target decreases the occupied fraction. Thus, target turnover provides a suitable explanation for the limited duration of the antiplatelet effect of the irreversible binder aspirin.[1] Moreover, target synthesis and degradation can be regulated and can function as feedback mechanisms.[100-104] A high rate of target turnover can limit the impact of a decreasing dissociation rate constant and can increase the impact of the association rate constant (Figure 4).

\subsubsection{Signal transduction}

Apart from these multiple factors which influence the target occupancy profiles, another step is required to predict effect kinetics from target occupancy profiles. To do this, the kinetics of all signal transduction steps need to be taken into account. The significance of signal transduction kinetics with respect to binding kinetics has been indicated by a simulation study of binding kinetics, enzyme inhibition and several signal transduction pathways.[17] However, since signal transduction can have various mechanisms and includes feedback mechanisms, the influence of signal transduction on the role of drug-target binding kinetics can differ greatly between targets.

Although the kinetics of signal transduction can be important, direct relationships between target occupancy and drug effect have been characterized for a few targets. However, in vivo target occupancy and drug effect are rarely measured 

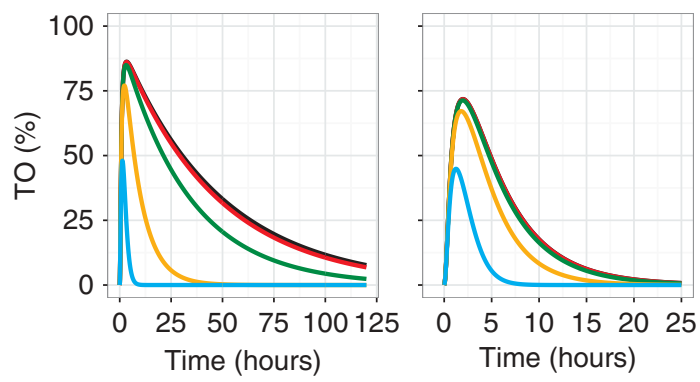

Figure 4. The influence of drug-target binding kinetics on target occupancy (TO) can be influenced by target turnover, as described by the model of Hong et al. for the antiplatelet effect of aspirin and ibuprofen.[1] The drug target dissociation rate $\left(k_{\text {off }}\right)$ changes from 0 (black line representing aspirin) to $0.001 \mathrm{~h}^{-1}$ (red), $0.01 \mathrm{~h}^{-1}$ (green), $0.1 \mathrm{~h}^{-1}$ (orange) and $1 \mathrm{~h}^{-1}$ (blue). The target degradation rate constant $\left(k_{\text {deg }}\right)$ is $0.02 \mathrm{~h}^{-1}$ (as identified for aspirin and ibuprofen) for the left panel and $0.2 \mathrm{~h}^{-1}$ for the right panel (note the different time scale). The target synthesis rate constant $\left(k_{\text {syn }}\right)$ was adjusted accordingly to obtain a steady-state target concentration of $25 \mathrm{nM}$ for both panels: $k_{\text {syn }}=25 \times k_{\text {deg. }}$.

simultaneously, and mathematical models are often required to estimate the relationship between target occupancy and effect from pharmacokinetic and pharmacodynamic data. One example where in vivo target occupancy and drug effect were measured simultaneously for the dopamine $\mathrm{D}_{2}$ receptor demonstrated the typical hyperbolical relationship.[105] However, linear relationships between target occupancy and effect have been used by mathematical models as well, for example to describe drug effect for calcium channel blockers and DPP-4 inhibitors.[20,26]

\subsubsection{Integrated mathematical modeling}

To incorporate all the processes as described above for the prediction of target occupancy and drug effect and to use these predictions for the selection of the best drug candidates, quantitative mathematical description and integration of all these processes is essential.

Mathematical models have made use of drug-target binding kinetics in the previous decades to describe and predict the time course of drug effect.[20-26] These mathematical models most often use differential equations to describe the rate with which concentrations change, rather than describing the absolute value of a concentration for any time point. The use of differential equations requires solving of the differential equations for each time profile and each initial value, but it also allows the integration of numerous processes in a relatively simple way. As an example, the decrease in drug concentration due to elimination is often described by an equation like Equation 2, where $d C / d t$ is the change in drug concentration per time unit, $C$ is the drug concentration and $k_{\mathrm{el}}$ is the elimination rate constant.

$$
\frac{d C}{d t}=-k_{\mathrm{el}} \times C
$$

Equation 2 means that if $k_{\mathrm{el}}=0.1 \mathrm{~min}^{-1}$, for example, the drug concentration decreases with $10 \%$ every minute (if you solve the equation by taking time steps of 1 minute). For compartmental models, differential equations are used to describe the concentration in each compartment, and each compartment is considered to be homogeneous. For example, if the distribution of a drug over the body is fast or limited, the concentration profile of a drug in plasma can often be described by a one-compartment model with absorption and elimination. Such a compartmental approach can be used to describe drug-target binding by adding one or more compartments, which represent the drug-target complex, and assuming homogeneous distribution of the target in one of the pharmacokinetic compartments. This approach has been used for the simulations of Figures 1-4, where binding was simulated from a single compartment for Figures 2 and 4, from a brain compartment for Figure 3 while a constant concentration was used for Figure 1. Although these simulations are based on simplifying assumptions such as homogeneity, they provide a conceptual insight in the impact of the described processes on the relation between drug-target binding kinetics and target occupancy.

A special field where drug-target binding kinetics are taken into account as standard practice is the field of targetmediated drug disposition (TMDD). TMDD describes the pharmacokinetics of drugs (mostly biologicals) which are distributed and eliminated predominantly when bound to their target. In this situation, binding kinetics are required to describe the drugs' pharmacokinetics, since the pharmacokinetics depend on the binding and the dissociation is often relatively slow.[106-109] Mathematical analysis of a TMDD model revealed that $k_{\text {on }}$ had a more pronounced impact on the maximal target occupancy than $k_{\text {off. }}[110]$ Another field where drug-target binding kinetics are commonly incorporated in mathematical models is in the analysis of PET data (see Table 3). In all these examples and in the simulations shown in Figures 1-4, mathematical models have demonstrated their potential to further our understanding of the role of drug-target binding kinetics in their complex physiological context.

\section{Conclusion}

On the basis of the sparse amount of available literature estimates for drug-target binding kinetics, no conclusions can be made on how well in vivo binding kinetics are reflected in in vitro experiments. Moreover, differences in conditions, methodology and analysis avoid the comparison of available in vitro and in vivo estimates in many cases.

Next to the relation between in vitro and in vivo estimates of binding kinetics, the relation between in vivo binding kinetics and in vivo target occupancy and effect kinetics is 
also uncertain. This relation can be influenced by pharmacokinetics, endogenous competition, target tissue diffusion, nonspecific binding, signal transduction and other factors. A quantitative integration by means of mathematical models can greatly enhance our understanding of the role of drugtarget binding kinetics in this context.

This implies that more scientific support is required for the rational selection and development of drug candidates on basis of in vitro estimates of drug-target binding kinetics.

\section{Expert opinion}

The aforementioned determinants of target occupancy are related to each other, and need to be taken into account in an integrated manner. The use of compartmental modeling, as applied commonly in $\mathrm{PK} / \mathrm{PD}$ modeling, is an important tool to facilitate the integration of all kinetic processes which are involved in the generation of drug effect (Figure 5). The value of such models for all stages of drug discovery and development is increasingly recognized.[111-113]

The feasibility of such an integrative approach in (the early phases of) drug discovery and development may seem limited by its complex and time-consuming nature. However, it is important to note that some of these kinetic processes are system-specific processes (e.g. endogenous competition, target turnover and signal transduction). These system-specific processes are equal for all new compounds and will not decrease the screening throughput in drug discovery. The integrated analysis of these systemspecific processes can thus be used to define which drugspecific kinetic parameters (e.g. drug-target association and dissociation rates, nonspecific binding rates and pharmacokinetic parameters) are most relevant per target/drug class to screen for. During the drug discovery process, the screening can start with only the most important parameter and be gradually extended to the other relevant parameters to refine the compound selection. The integrated analysis of all contributors to drug effect will not only improve compound selection, but it will also enable drug-candidate optimization on the most relevant parameters and optimization of drug dosing and sampling times in (pre)clinical investigations.

\subsection{Context-dependency of binding kinetics values}

To enable the integrated analysis of the kinetic processes which determine a drug's effect kinetics, specific information on all of these separate processes is required. This urges the performance of both in vitro and in vivo experiments which deliver drug- and system-specific parameters for all kinetic processes. This is necessary to avoid experiments which inform only on the combined effect of multiple processes and thus deliver context-dependent information. For example, if an in vivo binding study is analyzed to determine only the rate with which the target occupancy inclines and declines after a certain dose, process-specific information is lacking because no specific information is collected about the pharmacokinetics or binding kinetics. This applies also to in vitro experiments. If a washout experiment is used to estimate the dissociation rate without a competing ligand, the obtained estimate can be a combined parameter for both dissociation and diffusion, because "rebinding" can occur. [97] The occurrence of multiple kinetic processes during one experiment is not necessarily problematic, as long as the results can be analyzed in a process-specific manner to enable optimal translation to different experimental or clinical conditions. This type of process-specific analysis can be obtained by using physiologically based PK/PD models with process-specific parameters.

\subsection{Need for an integrated approach}

Although experiments are available and in use to estimate the rate constants of the aforementioned kinetic processes, there is hardly any information available about what the

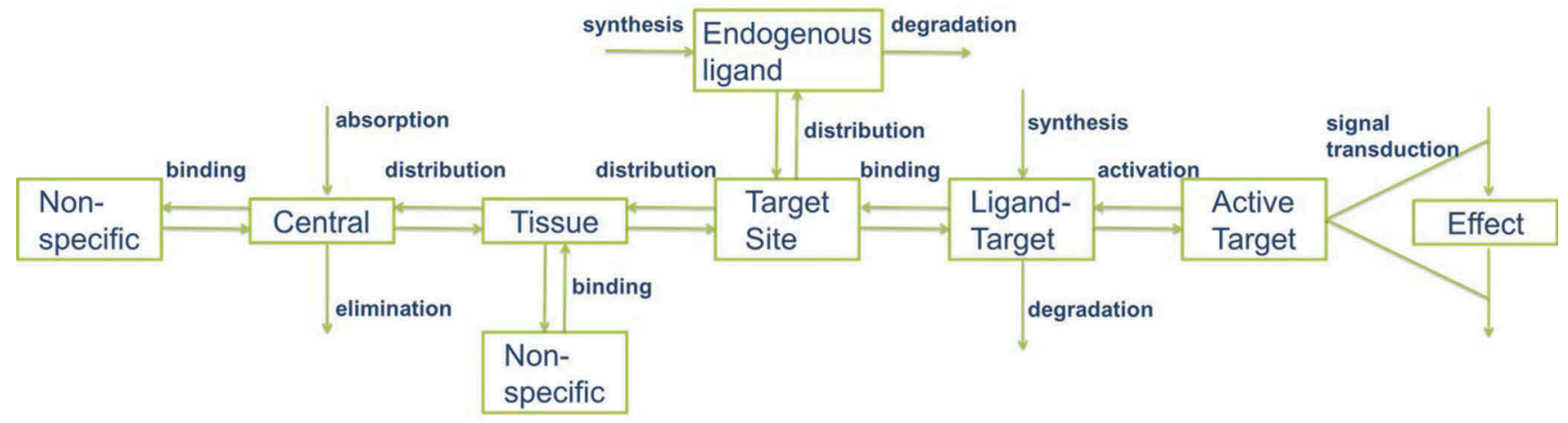

Figure 5. Schematic representation of the interconnected kinetic processes which determine target occupancy and effect kinetics. The central compartment represents the blood, the target site compartment represents the direct environment around the target, from where binding occurs. 
relative contribution of each of those processes is in the determination of target occupancy profiles during the various scenarios of drug treatment. To enable the prediction of in vivo target occupancy and effect profiles, integrated analysis of experimental data and increased theoretical insight in the role of all contributors to target occupancy and effect are required. Mathematical models which describe the mechanisms of all relevant processes can be of great value to both analyze experimental data and simulate various cases of drug treatment in a comprehensive and integrated fashion. Increasing knowledge of the drivers of drug effect is of critical importance to select the best drug candidates in drug discovery, to optimize drug therapy in drug development and improve the health of those in need of medicines.

\section{Declaration of interest}

The authors are part of the K4DD (Kinetics for Drug Discovery) consortium which is supported by the Innovative Medicines Initiative Joint Undertaking (IMI JU) under grant agreement no 115366 . The IMIJU is a project supported by the European Union's Seventh Framework Programme (FP7/ 2007-2013) and the European Federation of Pharmaceutical Industries and Associations (EFPIA). The authors are also supported by Leiden University. Furthermore, R Gilissen is an employee of Janssen Pharmaceuticals. The authors have no other relevant affiliations or financial involvement with any organization or entity with a financial interest in or financial conflict with the subject matter or materials discussed in the manuscript apart from those disclosed.

\section{References}

1. Hong Y, Gengo FM, Rainka MM, et al. Population pharmacodynamic modelling of aspirin- and ibuprofen-induced inhibition of platelet aggregation in healthy subjects. Clin Pharmacokinet. 2008;47 (2):129-137.

2. Katashima M, Yamamoto K, Tokuma Y, et al. Comparative pharmacokinetic/pharmacodynamic analysis of proton pump inhibitors omeprazole, lansoprazole and pantoprazole, in humans. Eur J Drug Metab Pharmacokinet. 1998;23(1):19-26.

3. Äbelö A, Holstein B, Eriksson UG, et al. Gastric acid secretion in the dog: a mechanism-based pharmacodynamic model for histamine stimulation and irreversible inhibition by omeprazole. J Pharmacokinet Pharmacodyn. 2002;29 (4):365-382.

4. Copeland RA. The dynamics of drug-target interactions: drug-target residence time and its impact on efficacy and safety. Expert Opin Drug Discov. 2010;5(4):305310.

5. Tummino PJ, Copeland RA. Residence time of receptor-ligand complexes and its effect on biological function. Biochemistry. 2008;47(20):5481-5492.

6. Swinney DC. The role of binding kinetics in therapeutically useful drug action. Curr Opin Drug Discov Devel. 2009;12(1):31-39.

7. Kinzer-Ursem TL, Linderman JJ. Both ligand- and cell-specific parameters control ligand agonism in a kinetic model of $\mathrm{g}$ protein-coupled receptor signaling. PLoS Comput Biol. 2007;3(1):84-94.

* This model shows the influence of the receptor environment on agonism.
8. Leysen JE, Gomeren W. Different kinetic properties of neuroleptic receptor binding in the rat striatum and frontal cortex. Life Sci. 1978;23(5):447-452.

9. Perry DC, Mullis KB, Oie S, et al. Opiate antagonist receptor binding in vivo: evidence for a new receptor binding model. Brain Res. 1980;199(1):49-61.

* This paper is one of the few examples where diffusion-limited binding was identified in vivo.

10. Syrota A, Paillotin G, Davy JM, et al. Kinetics of in vivo binding of antagonist to muscarinic cholinergic receptor in the human heart studied by positron emission tomography. Life Sci. 1984;35(9):937945.

11. Guo D, Xia L, Van Veldhoven JPD, et al. Binding Kinetics of ZM241385 derivatives at the human adenosine $\mathrm{A} 2 \mathrm{~A}$ receptor. ChemMedChem. 2014;9(4):752-761.

12. Schneider EV, Böttcher J, Huber R, et al. Structure-kinetic relationship study of CDK8/CycC specific compounds. Proc Natl Acad Sci USA. 2013;110(20):80818086.

13. Copeland RA, Pompliano DL, Meek TD. Drug-target residence time and its implications for lead optimization. Nat Rev Drug Discov. 2006;5(9):730-739.

14. Vauquelin G, Van Liefde I. Slow antagonist dissociation and long-lasting in vivo receptor protection. Trends Pharmacol Sci. 2006;27(7):356-359.

15. Dahl G, Akerud T. Pharmacokinetics and the drug-target residence time concept. Drug Discov Today. 2013;18(1516):697-707.
* This paper demonstrates the impact of drug-target binding kinetics in a simple model of in vivo pharmacokinetics and target binding.

16. Guo D, Hillger JM, IJzerman AP, et al. Drug-target residence time: a case for $\mathrm{G}$ protein-coupled receptors. Med Res Rev. 2014;34(4):856-892.

17. Yin N, Pei J, Lai L. A comprehensive analysis of the influence of drug binding kinetics on drug action at molecular and systems levels. Mol Biosyst. 2013;9 (6):1381-1389.

18. Vauquelin G, Bostoen S, Vanderheyden P, et al. Clozapine, atypical antipsychotics, and the benefits of fast-off D2 dopamine receptor antagonism. Naunyn Schmiedebergs Arch Pharmacol. 2012;385 (4):337-372.

** This paper provides comprehensive simulations on the impact of binding kinetics on dopamine antagonism.

19. Vauquelin G. Rebinding: or why drugs may act longer in vivo than expected from their in vitro target residence time. Expert Opin Drug Discov. 2010;5 (10):927-941.

* This paper explores the influence of diffusion-limited binding on the impact of drug-target binding kinetics on target occupancy.

20. Shimada S, Nakajima Y, Yamamoto K, et al. Comparative pharmacodynamics of eight calcium channel blocking agents in Japanese essential hypertensive patients. Biol Pharm Bull. 1996;19(3):430-437.

** This paper provides one of the few examples where drug-target binding 
kinetics have been estimated in vivo for a series of drugs for the same target.

21. Yun H-Y, Yun M-H, Kang W, et al. Pharmacokinetics and pharmacodynamics of benidipine using a slow receptor-binding model. J Clin Pharm Ther. 2005;30 (6):541-547.

22. Steagall PVM, Pelligand L, Giordano T, et al. Pharmacokinetic and pharmacodynamic modelling of intravenous, intramuscular and subcutaneous buprenorphine in conscious cats. Vet Anaesth Analg. 2013;40(1):83-95.

23. Ramsey SJ, Attkins NJ, Fish R, et al. Quantitative pharmacological analysis of antagonist binding kinetics at CRF1 receptors in vitro and in vivo. $\mathrm{Br} \mathrm{J}$ Pharmacol. 2011;164(3):992-1007.

24. Yassen A, Olofsen E, Dahan A, et al. Pharmacokinetic-pharmacodynamic modeling of the antinociceptive effect of buprenorphine and fentanyl in rats : role of receptor equilibration kinetics. J Pharmacol Exp Ther. 2005;313(3):11361149.

25. Äbelö A, Andersson M, Holmberg AA, et al. Application of a combined effect compartment and binding model for gastric acid inhibition of AR-HO47108: a potassium competitive acid blocker, and its active metabolite AR-HO47116 in the dog. Eur J Pharm Sci. 2006;29(2):91-101.

26. Landersdorfer CB, He YL, Jusko WJ. Mechanism-based population pharmacokinetic modelling in diabetes: Vildagliptin as a tight binding inhibitor and substrate of dipeptidyl peptidase IV. Br J Clin Pharmacol. 2012;73(3):391-401.

27. Guo D, Mulder-Krieger T, IJzerman AP, et al. Functional efficacy of adenosine A2A receptor agonists is positively correlated to their receptor residence time. $\mathrm{Br} \mathrm{J}$ Pharmacol. 2012;166(6):1846-1859.

28. Motulsky HJ, Mahan LC. The kinetics of competitive radioligand binding predicted mass action by the law of mass action. Mol Pharmacol. 1984;25(1):1-9.

29. Zweemer AJM, Nederpelt I, Vrieling H, et al. Multiple binding sites for smallmolecule antagonists at the CC chemokine receptor 2. Mol Pharmacol. 2013;84 (4):551-561.

30. Guo D, Van Dorp EJH, Mulder-Krieger T, et al. Dual-point competition association assay: a fast and high-throughput kinetic screening method for assessing ligand-receptor binding kinetics. J Biomol Screen. 2013;18(3):309-320.

31. Mathis G, Pierre N, Tardieu J Determination of association(kon) and dissociation(koff) rate constants of spiperone on the dopamine D2 receptor using the Tag-lite ${ }^{\circledR}$ platform. 2011. p. SBS 17th Annual Conference. Orlando, USA

32. Schiele F, Ayaz P, Fernández-Montalván A. A universal homogeneous assay for high-throughput determination of binding kinetics. Anal Biochem. 2015;468:42-49.

33. Nederpelt I, Schuldt V, Schiele F, et al. Characterization of $12 \mathrm{GnRH}$ peptide agonists - a kinetic perspective. Br J Pharmacol. 2015; Epub ahead of print. doi: 10.1111/bph.13342.

34. Patching SG. Surface plasmon resonance spectroscopy for characterisation of membrane protein-ligand interactions and its potential for drug discovery. Biochim Biophys Acta. 2014;1838(1):43-55.

35. Rich RL, Myszka DG. Higher-throughput, label-free, real-time molecular interaction analysis. Anal Biochem. 2007;361 (1):1-6.

36. Shiau AK, Massari ME, Ozbal CC. Back to basics: label-free technologies for small molecule screening. Comb Chem High Throughput Screen. 2008;11(3):231-237.

37. Gronewold TMA, Baumgartner A, Hierer $\mathrm{J}$, et al. Kinetic binding analysis of aptamers targeting HIV-1 proteins by a combination of a microbalance array and mass spectrometry (MAMS). J Proteome Res. 2009;8(7):3568-3577.

38. Gronewold TMA. Surface acoustic wave sensors in the bioanalytical field: recent trends and challenges. Anal Chim Acta. 2007;603(2):119-128.

39. Kenakin T, Jenkinson S, Watson C. Determining the potency and molecular mechanism of action of insurmountable antagonists. J Pharmacol Exp Ther. 2006;319(2):710-723.

40. Vauquelin G, Fierens F, Verheijen I, et al. Insurmountable AT 1 receptor antagonism: the need for different antagonist binding states of the receptor glutamate signalling in the lung. Trends Pharmacol Sci. 2001;22(7):343-344.

41. Szczuka A, Wennerberg M, Packeu A, et al. Molecular mechanisms for the persistent bronchodilatory effect of the beta 2-adrenoceptor agonist salmeterol. Br J Pharmacol. 2009;158(1):183-194.
42. Lindström E, Von Mentzer B, Påhlman I, et al. Neurokinin 1 receptor antagonists: correlation between in vitro receptor interaction and in vivo efficacy. J Pharmacol Exp Ther. 2007;322(3):12861293.

43. Le MT, Pugsley MK, Vauquelin G, et al. Molecular characterisation of the interactions between olmesartan and telmisartan and the human angiotensin II AT1 receptor. Br J Pharmacol. 2007;151(7):952-962.

44. Atack JR, Wong DF, Fryer TD, et al. Benzodiazepine binding site occupancy by the novel GABAA receptor subtype-selective drug 7-(1,1-dimethylethyl)-6-(2-ethyl2H-1,2,4-triazol-3-ylmethoxy)-3-(2-fluorophenyl)-1,2,4-triazolo[4,3-b]pyridazine (TPA023) in rats, primates, and humans. J Pharmacol Exp Ther. 2010;332(1):17-25.

45. Laruelle M. Measuring dopamine synaptic transmission with molecular imaging and pharmacological challenges: the state of the art. Neuromethods. 2012;71:163-203.

46. Chernet E, Martin LJ, Li D, et al. Use of LC/MS to assess brain tracer distribution in preclinical, in vivo receptor occupancy studies: dopamine D2, serotonin $2 \mathrm{~A}$ and NK-1 receptors as examples. Life Sci. 2005;78(4):340-346.

47. Barth VN. Typical and atypical antipsychotics: relationships between rat in vivo dopamine $\mathrm{D}(2)$ receptor occupancy assessed using LC/MS and changes in neurochemistry and catalepsy. Section Title: Mammalian Hormones. Ann Arbor, MI: ProQuest Information Learning Company;2006.

48. Need AB, McKinzie JH, Mitch $\mathrm{CH}$, et al. In vivo rat brain opioid receptor binding of LY255582 assessed with a novel method using LC/MS/MS and the administration of three tracers simultaneously. Life Sci. 2007;81(17-18):1389-1396.

49. Hume SP, Gunn RN, Jones T. Pharmacological constraints associated with positron emission tomographic scanning of small laboratory animals. Eur J Nucl Med. 1998;25(2):173-176.

50. Kung M-P, Kung HF. Mass effect of injected dose in small rodent imaging by SPECT and PET. Nucl Med Biol. 2005;32(7):673-678.

51. Hume SP, Myers R, Bloomfield PM, et al. Quantitation of carbon-11-labeled raclopride in rat striatum using positron emission tomography. Synapse. 1992;12 (1):47-54. 
52. Tantawy MN, Jones CK, Baldwin RM, et al. [(18)F]Fallypride dopamine D2 receptor studies using delayed microPET scans and a modified Logan plot. Nucl Med Biol. 2009;36(8):931-940.

53. Lancelot S, Zimmer L. Small-animal positron emission tomography as a tool for neuropharmacology. Trends Pharmacol Sci. 2010;31(9):411-417.

54. Müller CP, Pum ME, Amato D, et al. The in vivo neurochemistry of the brain during general anesthesia. J Neurochem. 2011;119(3):419-446.

55. Schiffer WK, Mirrione MM, Biegon A, et al. Serial microPET measures of the metabolic reaction to a microdialysis probe implant. J Neurosci Methods. 2006;155 (2):272-284.

56. Ginovart N, Sun W, Wilson AA, et al. Quantitative validation of an intracerebral beta-sensitive microprobe system to determine in vivo drug-induced receptor occupancy using [11C]Raclopride in rats. Synapse. 2004;52(2):89-99.

57. Balasse L, Maerk J, Pain F, et al. PIXSIC, a pixelated $\beta(+)$-sensitive probe for radiopharmacological investigations in rat brain: binding studies with [(18)F]MPPF. Mol Imaging Biol. 2015;17(2):163-167.

58. Polikov VS, Tresco PA, Reichert WM. Response of brain tissue to chronically implanted neural electrodes. J Neurosci Methods. 2005;148(1):1-18.

59. Wang Y, Michael AC. Microdialysis probes alter presynaptic regulation of dopamine terminals in rat striatum. J Neurosci Methods. 2012;208(1):34-39.

60. Märk J, Benoit D, Balasse L, et al. A wireless beta-microprobe based on pixelated silicon for in vivo brain studies in freely moving rats. Phys Med Biol. 2013;58(13):4483-4500.

61. Li J, Fish RL, Cook SM, et al. Comparison of in vivo and ex vivo [3H] flumazenil binding assays to determine occupancy at the benzodiazepine binding site of rat brain GABAA receptors. Neuropharmacology. 2006;51(1):168172.

62. Schotte A, Janssen PF, Gommeren W, et al. Risperidone compared with new and reference antipsychotic drugs: in vitro and in vivo receptor binding. Psychopharmacology (Berl). 1996;124(12):57-73.

63. Lengyel K, Pieschl R, Strong T, et al. Ex vivo assessment of binding site occupancy of monoamine reuptake inhibitors: methodology and biological significance. Neuropharmacology. 2008;55(1):63-70.

64. Langlois X, Te Riele P, Wintmolders C, et al. Use of the beta-imager for rapid ex vivo autoradiography exemplified with central nervous system penetrating neurokinin 3 antagonists. J Pharmacol Exp Ther. 2001;299(2):712-717.

65. Baker JG, Hall IP, Hill SJ. Pharmacological characterization of CGP 12177 at the human beta(2)-adrenoceptor. Br J Pharmacol. 2002;137(3):400-408.

66. Delforge J, Syrota A, Lançon JP, et al. Cardiac beta-adrenergic receptor density measured in vivo using PET, CGP 12177 , and a new graphical method. J Nucl Med. 1991;32(4):739-748.

67. Kessler RM, Votaw JR, Schmidt DE, et al. High affinity dopamine D2 receptor radioligands. 3.[123I] and [125I]epidepride: in vivo studies in rhesus monkey brain and comparison with in vitro pharmacokinetics in rat brain. Life Sci. 1993;53(3):241-250.

68. Mukherjee J, Yang ZY, Brown T, et al. 18F-desmethoxyfallypride: a fluorine-18 labeled radiotracer with properties similar to carbon-11 raclopride for PET imaging studies of dopamine D2 receptors. Life Sci. 1996;59(8):669-678.

69. Mukherjee J, Yang Z-Y, Lew R, et al. Evaluation of $\mathrm{d}$-Amphetamine effects on the binding of dopamine D-2 receptor radioligand, $18 \mathrm{~F}$-fallypride in nonhuman primates using positron emission tomography. Synapse. 1997;27(1):1-13.

70. Christian BT, Narayanan T, Shi B, et al. Measuring the in vivo binding parameters of [18F]-fallypride in monkeys using a PET multiple-injection protocol. J Cereb Blood Flow Metab. 2004;24(3):309-322.

71. Vandehey NT, Moirano JM, Converse AK, et al. High-affinity dopamine D2/ D3 PET radioligands 18F-fallypride and 11C-FLB457: a comparison of kinetics in extrastriatal regions using a multipleinjection protocol. J Cereb Blood Flow Metab. 2010;30(5):994-1007.

72. Leysen JE, Gommeren W. Drug-receptor dissociation time, new tool for drug research: receptor binding affinity and drug-receptor dissociation profiles of serotonin-S2, dopamine-D2, histamine-HI antagonists, and opiates. Drug Dev Res. 1986;131:119-131.

73. Perlmutter JS, Kilbourn MR, Welch MJ, et al. Non-steady-state measurement of in vivo receptor binding with positron emission tomography :"Dose Response" analysis. J Neurosci. 1989;9(7):2344-2352.

74. Leslie CA, Bennett JP. [3H]spiperone binds selectively to rat striatal D2 dopamine receptors in vivo: a kinetic and pharmacological analysis. Brain Res. 1987;407(2):253-262.

75. Kapur S, Seeman P. Antipsychotic agents differ in how fast they come off the dopamine D2 receptors. Implications for atypical antipsychotic action. J Psychiatry Neurosci. 2000;25(2):161-166.

76. Johnson M, Kozielska M, Pilla Reddy V, et al. Mechanism-based pharmacokineticpharmacodynamic modeling of the dopamine D2 receptor occupancy of olanzapine in rats. Pharm Res. 2011;28(10):24902504.

77. Laruelle M, Abi-Dargham A, Ai-Tikriti MS, et al. SPECT quantification of [123I] lomazenil binding to benzodiazepine receptors in nonhuman primates: II. Equilibrium analysis of constant infusion experiments and correlation with in vitro parameters. J Cereb Blood Flow Metab. 1994;14(3):453-465.

78. Millet P, Graf C, Moulin M, et al. SPECT quantification of benzodiazepine receptor concentration using a dual-ligand approach. J Nucl Med. 2006;47(5):783792.

79. Sakiyama Y, Saito M, Inoue O. Acute treatment with pentobarbital alters the kinetics of in vivo receptor binding in the mouse brain. Nucl Med Biol. 2006;33 (4):535-541.

80. Sihver W, Sihver S, Bergström M, et al. Aspects for in vitro characterization of receptor ligands: receptor binding using 11C-labeled A detailed study with the benzodiazepine receptor antagonist [11C] Ro 15-1788. Nucl Med Biol. 1997;24 (8):723-731.

81. Koeppe RA, Holthoff VA, Frey KA, et al. Compartmental analysis of [11C]flumazenil kinetics for the estimation of ligand transport rate and receptor distribution using positron emission tomography. J Cereb Blood Flow Metab. 1991;11 (5):735-744.

82. Ishii A, Toyama J. Binding properties of $( \pm)[3 \mathrm{H}]$ benidipine hydrochloride to rat heart membranes. J Cardiovasc Pharmacol. 1993;21(2):191-196.

83. Cassel JA, Daubert JD, DeHaven RN. [(3) $\mathrm{H}]$ Alvimopan binding to the mu opioid 
receptor: comparative binding kinetics of opioid antagonists. Eur J Pharmacol. 2005;520(1-3):29-36.

84. Yassen A, Olofsen E, Romberg R, et al. Mechanism-based PK/PD modeling of the respiratory depressant effect of buprenorphine and fentanyl in healthy volunteers. Clin Pharmacol Ther. 2007;81(1):50-58.

85. Yassen A, Kan J, Olofsen E, et al.. Mechanism-based pharmacokinetic-pharmacodynamic modeling of the respiratorydepressant effect of buprenorphine and fentanyl in rats. J Pharmacol Exp Ther. 2006;319(2):682-692.

86. Casadó V, Allende G, Mallol J, et al. Thermodynamic analysis of agonist and antagonist binding to membrane-bound and solubilized A1 adenosine receptors. J Pharmacol Exp Ther. 1993;266(3):14631474.

87. Langlois X, Megens A, Lavreysen H, et al. Pharmacology of JNJ-37822681, a specific and fast-dissociating D2 antagonist for the treatment of schizophrenia. J Pharmacol Exp Ther. 2012;342(1):91-105.

88. Treherne JM, Young JM. Temperaturedependence of the kinetics of the binding the histamine $\mathrm{H} 1$-receptor: comparison with the kinetics of $[3 \mathrm{H}]$-mepyramine. $\mathrm{Br}$ J Pharmacol. 1988;94(3):811-822.

89. Wallace RM, Young JM. Temperature dependence related compounds of the binding of $[3 \mathrm{H}]$ Mepyramine to the Histamine H1 Receptor. Mol Pharmacol. 1983;23(1):60-66.

90. Sakai S. Effect of hormones on dissociation of prolactin from the rabbit mammary gland prolactin receptor. Biochem J. 1991;279:461-465.

91. Endres CJ, Kolachana BS, Saunders RC, et al. Kinetic modeling of [11C]raclopride: combined PET-microdialysis studies. J Cereb Blood Flow Metab. 1997;17 (9):932-942.

92. Morris ED, Yoder KK. Positron emission tomography displacement sensitivity: predicting binding potential change for positron emission tomography tracers based on their kinetic characteristics. J Cereb Blood Flow Metab. 2007;27 (3):606-617.

93. Kapur S, Seeman P. Does fast dissociation from the dopamine D2 receptor explain the action of atypical antipsychotics?: a new hypothesis. Am J Psychiatry. 2001;158(3):360-369.
94. Wiley HS. Anomalous binding of epidermal growth factor to A431 cells is due to the effect of high receptor densities and a saturable endocytic system. J Cell Biol. 1988;107(2):801-810.

95. Packeu A, Wennerberg M, Balendran A, et al. Estimation of the dissociation rate of unlabelled ligand-receptor complexes by a "two-step" competition binding approach. Br J Pharmacol. 2010;161(6):1311-1328.

96. Vauquelin G, Van Liefde I. Radioligand dissociation measurements: potential interference of rebinding and allosteric mechanisms and physiological relevance of the biological model systems. Expert Opin Drug Discov. 2012;7(7):583-595.

97. Vauquelin G, Charlton SJ. Long-lasting target binding and rebinding as mechanisms to prolong in vivo drug action. $\mathrm{Br}$ Pharmacol. 2010;161(3):488-508.

98. Peletier LA, Benson N. van der Graaf PH. Impact of protein binding on receptor occupancy: a two-compartment model. J Theor Biol. 2010;265(4):657-671.

* This paper provides insight into the possible impact of non-specific binding on target occupancy.

99. Proost JH, Wierda JM, Meijer DK. An extended pharmacokinetic/pharmacodynamic model describing quantitatively the influence of plasma protein binding, tissue binding, and receptor binding on the potency and time course of action of drugs. J Pharmacokinet Biopharm. 1996;24(1):45-77.

100. Kim KM, Valenzano KJ, Robinson SR, et al. Differential regulation of the dopamine D2 and D3 receptors by G proteincoupled receptor kinases and beta-arrestins. J Biol Chem. 2001;276(40):3740937414.

101. Macey TA, Gurevich VV, Neve KA. Preferential interaction between the dopamine D2 receptor and arrestin2 in neostriatal neurons. Mol Pharmacol. 2004;66 (6):1635-1642.

102. Paspalas CD, Rakic P, Goldman-Rakic PS. Internalization of $\mathrm{D} 2$ dopamine receptors is clathrin-dependent and select to dendroaxonic appositions in primate prefrontal cortex. Eur J Neurosci. 2006;24(5):1395-1403.

103. Dang VC, Christie MJ. Mechanisms of rapid opioid receptor desensitization, resensitization and tolerance in brain neurons. Br J Pharmacol. 2012;165(6):17041716.
104. Skinbjerg M, Liow J-S, Seneca N, et al. D2 dopamine receptor internalization prolongs the decrease of radioligand binding after amphetamine: a PET study in a receptor internalization-deficient mouse model. Neuroimage. 2010;50(4):14021407.

105. Haraguchi $\mathrm{K}$, Ito $\mathrm{K}$, Kotaki $\mathrm{H}$, et al. Prediction of drug-induced catalepsy based on dopamine D1, D2, and muscarinic acetylcholine receptor occupancies. Drug Metab Dispos. 1997;25(6):675-684.

106. Mager DE, Jusko WJ. General pharmacokinetic model for drugs exhibiting targetmediated drug disposition. J Pharmacokinet Pharmacodyn. 2001;28 (6):507-532.

107. Eppler SM, Combs DL, Henry TD, et al. A target-mediated model to describe the pharmacokinetics and hemodynamic effects of recombinant human vascular endothelial growth factor in humans. Clin Pharmacol Ther. 2002;72(1):20-32.

108. Jin F, Krzyzanski W. Pharmacokinetic model of target-mediated disposition of thrombopoietin. Aaps J. 2004;6(1):86-93.

109. Retlich S, Duval V, Graefe-Mody U, et al. Impact of target-mediated drug disposition on Linagliptin pharmacokinetics and DPP-4 inhibition in type 2 diabetic patients. J Clin Pharmacol. 2010;50 (8):873-885.

110. Aston PJ, Derks G, Raji A, et al. Mathematical analysis of the pharmacokinetic-pharmacodynamic (PKPD) behaviour of monoclonal antibodies: predicting in vivo potency. J Theor Biol. 2011;281 (1):113-121.

111. Zhang L, Sinha V, Forgue ST, et al. Model-based drug development: the road to quantitative pharmacology. J

Pharmacokinet Pharmacodyn. 2006;33 (3):369-393.

112. Stone JA, Banfield C, Pfister $\mathrm{M}$, et al. Model-based drug development survey finds pharmacometrics impacting decision making in the pharmaceutical industry. J Clin Pharmacol. 2010;50(S9):20S- 30S.

113. Van Der Graaf PH, Gabrielsson J. Pharmacokinetic - pharmacodynamic reasoning in drug discovery and early development. Future Med Chem. 2009;1 (8):1371-1374.

\section{Affiliation}

Wilhelmus EA de Witte ${ }^{1}$, Yin Cheong Wong ${ }^{1}$, Indira Nederpelt ${ }^{2}$, Laura $\mathrm{H}$ Heitman ${ }^{2}$, Meindert 
Rational use of in vitro drug-target binding kinetics

Danhof ${ }^{1}$, Piet $\mathrm{H}$ van der $\mathrm{Graaf}^{1}$, Ron AHJ Gilissen $^{3}$ \& Elizabeth C.M. de Lange ${ }^{\dagger 1}$

${ }^{\dagger}$ Author for correspondence

${ }^{1}$ Division of Pharmacology, Leiden Academic Centre for Drug Research, Leiden University,
Einsteinweg 55, 2333 CC Leiden, The Netherlands

E-mail: ecmdelange@lacdr.leidenuniv.nl ${ }^{2}$ Division of Medicinal Chemistry, Leiden Academic Centre for Drug Research, Leiden
University, Einsteinweg 55, 2333 CC Leiden, The Netherlands

${ }^{3}$ A Division of Janssen Pharmaceutica N.V., Janssen Research and Development,

Turnhoutseweg 30, Beerse 2340, Belgium 